\title{
PRODUÇÃO DE PAINÉIS AGLOMERAdOS HOMOGÊNEOS E MULTICAMADAS DE Melia azedarach (CINAMOMO) E Pinus taeda COM DIFERENTES TEORES DE RESINA
}

\author{
Setsuo Iwakiri ${ }^{1}$, Jorge Luis Monteiro de Matos ${ }^{1}$, Rosilani Trianoski², José Guilherme Prata ${ }^{3}$
}

(recebido: 28 de outubro de 2010; aceito: 30 de março de 2012)

RESUMO: Nesta pesquisa, objetivou-se avaliar a qualidade de painéis aglomerados homogêneos e multicamadas produzidos com madeira de Melia azedarach e Pinus taeda com diferentes teores de resina. Foram produzidos painéis experimentais com densidade nominal de $0,75 \mathrm{~g} / \mathrm{cm}^{3}$, utilizando a resina ureia-formaldeído na proporção de $6 \%$ e $8 \%$ (sólido resinoso - base peso seco das partículas) na camada interna e de $8 \%$ e $10 \%$ nas camadas externas. Os painéis foram prensados com pressão específica de $40 \mathrm{kgf} / \mathrm{cm}^{2}$, temperatura de $160^{\circ} \mathrm{C}$ e tempo de prensagem de 8 minutos. As avaliações das propriedades físicas e mecânicas dos painéis indicaram um grande potencial da espécie Melia azedarach para a produção de painéis aglomerados. O aumento no teor de resina das camadas interna e externa melhoram as propriedades de absorção de água e inchamento em espessura. Entretanto, as propriedades mecânicas não foram afetadas.

Palavras-chave: Painéis homogêneos e multicamadas, espécie alternativa, teor de resina, resina ureia-formaldeído.

\section{PRODUCTION OF HOMOGENEOUS AND MULTILAYER PARTICLEBOARD FROM Melia azedarach (CINAMOMO) AND Pinus taeda WITH DIFFERENT RESIN CONTENTS}

ABSTRACT: This research was developed to evaluate the quality of homogeneous and multilayer particleboards manufactured from Melia azedarach wood and Pinus taeda with different resin contents. The experimental boards were manufactured with the nominal density of $0,75 \mathrm{~g} / \mathrm{cm}^{3}$, using the urea-formaldehyde resin in the proportion of $6 \%$ and $8 \%$ (solid content based on oven dried wood particles) in the internal layer and $8 \%$ and $10 \%$ in the external layers. The boards were pressed at the specific pressure of $40 \mathrm{kgf} / \mathrm{cm}^{2}$, temperature of $160^{\circ} \mathrm{C}$ and press time of 8 minutes. The evaluations of the physical and mechanical properties of the boards showed the high potentiality of Melia azedarach for particleboard manufacture. The increase of resin content in the internal and external layers improved the properties of water absorption and thickness swelling. However, the mechanical properties were not affected.

Key words: Homogeneous and multilayer panels, alternative species, resin content, urea-formaldehyde.

\section{INTRODUÇÃO}

A base florestal brasileira que sustenta as indústrias de painéis reconstituídos de madeira é constituída de pinus e eucalipto, provenientes de plantios florestais localizados nas regiões sul e sudeste do país. Nas últimas duas décadas, as indústrias do setor de painéis reconstituídos de madeira têm empreendido grandes investimentos na implantação de novas unidades produtivas, além do aumento em novas áreas de plantios florestais para assegurar o suprimento de madeira. De acordo com o estudo setorial do Banco Nacional de Desenvolvimento Econômico e Social - BNDES (2008), a produção brasileira de painéis aglomerados cresceu de 0,866 milhões de m³/ano em 1995 para 2,098 milhões de $\mathrm{m}^{3}$ em 2005 . O crescimento médio nesse período foi de $9,3 \%$ ao ano, sendo um percentual bem superior ao 4,5\% do nível mundial. Esse crescimento deve-se à demanda crescente do setor moveleiro que utiliza amplamente esse produto na fabricação de móveis.

Para reduzir as dependências de madeiras de pinus e eucalipto, o setor de base florestal tem investido em pesquisas nas áreas de silvicultura e tecnologia da madeira em busca de espécies alternativas de rápido crescimento para a produção de madeira. Entre as inúmeras espécies em estudo, a Melia azedarach, conhecida como Cinamomo, apresenta características promissoras em termos de crescimento e produção de madeira. A madeira de Cinamomo apresenta semelhança com a madeira de cedro e sua massa específica aparente média é em torno de 0,43 $\mathrm{g} / \mathrm{cm}^{3}$. Entre as inúmeras aplicações do Cinamomo, podese mencionar o uso na fabricação de móveis e esquadrias, trabalhos de marcenaria, madeira serrada, laminados e faqueados (CENTRO AGRONÔMICO TROPICAL DE IVESTIGACION Y ENSEÑANZA - CATIE, 1986).

\footnotetext{
${ }^{1}$ Engenheiro Florestal, Professor Doutor em Engenharia Florestal - Unviersidade Federal do Paraná/UFPR - Departamento de Engenharia e Tecnologia Florestal-80210-170 - Curitiba, PR, Brasil - setsuo@ufpr.br, jmatos@ufpr.br

${ }^{2}$ Engenheira Industrial Madeireira, Doutoranda em Engenharia Florestal - Universidade Federal do Paraná/UFPR - Programa de Pós-graduação em Engenharia Florestal - 80210-170 - Curitiba, PR - rosillani@gmail.com

${ }^{3}$ Engenheiro Florestal, Professor Doutor em Engenharia Florestal - Universidade Federal do Paraná/UFPR - Departamento de Engenharia e Tecnologia Florestal - 80210-170 - Curitiba, PR - jgprata@ufpr.br
}

Cerne, Lavras, v. 18, n. 3, p. 465-470, jul./set. 2012 
De acordo com Venson (2003), por essa espécie apresentar propriedades mecânicas baixas, o Cinamomo não é recomendado para uso estrutural ou em aplicações que requeiram maior resistência, sendo recomendado utilizar essa madeira em produtos reconstituídos.

As espécies de madeira por apresentarem grande variabilidade na sua estrutura anatômica, propriedades físicas e químicas, podem exercer influências positivas e negativas na fabricação de produtos colados de madeira. Segundo Marra (1992), as propriedades da madeira possuem forte influência na formação da ligação adesiva e, geralmente, as madeiras de folhosas apresentam mais dificuldades do que as de coníferas.

A escolha de espécies de madeira para produção de painéis aglomerados deve ser baseada em alguns parâmetros importantes como a densidade, $\mathrm{pH}$ e extrativos (MALONEY, 1993; MOSLEMI, 1974; TSOUMIS, 1993). A densidade da madeira é um dos requisitos básicos na escolha de espécies para produção de painéis aglomerados, por influenciar na sua razão de compactação. Segundo os autores, a razão de compactação, que é a relação entre a densidade do painel e a densidade da madeira, deve ser de no mínimo 1,3 para assegurar uma área de contato satisfatória entre as partículas e densificação suficiente para a formação do painel. Moslemi (1974) afirma que, para painéis de mesma densidade, produzidos com madeira de baixa densidade, as suas propriedades mecânicas serão superiores, entretanto, a sua estabilidade dimensional será inferior em comparação aos painéis produzidos com madeira de maior densidade. Segundo Maloney (1993), espécies com densidade de até $0,55 \mathrm{~g} / \mathrm{cm}^{3}$ são as mais adequadas para produção de painéis de partículas por atingirem uma razão de compactação entre 1,3 e 1,6, considerada faixa ideal para o processo de densificação e consolidação do painel até a espessura final.

Com relação ao $\mathrm{pH}$ e extrativos presentes na madeira, Marra (1992) afirma que esses parâmetros podem ter influencia direta na cura da resina e, consequentemente, na qualidade dos painéis produzidos. $\mathrm{O} \mathrm{pH}$ da madeira pode variar entre 3,0 a 5,5 e, de acordo com Kelly (1977), madeiras com $\mathrm{pH}$ muito ácido podem causar a pré-cura da resina ureia-formaldeído, durante a fase de fechamento da prensa, prejudicando o grau de adesão entre as partículas e redução nos valores das propriedades dos painéis. A influência dos extrativos na polimerização e cura do adesivo é relatada por vários pesquisadores. Marra (1992) afirma que madeiras com elevados teores de extrativos apresentam dificuldades de colagem, resultando em baixa resistência da ligação adesiva entre as partículas.

Cerne, Lavras, v. 18, n. 3, p. 465-470, jul./set. 2012
Quanto aos parâmetros do processo produtivo, a quantidade de resina empregada na formação do painel aglomerado é um fator de impacto, tanto em termos de qualidade do painel, quanto ao seu custo final. Maloney (1993) e Moslemi (1974) afirmam que a quantidade de resina está diretamente associada à área superficial específica das partículas. Portanto, partículas menores da camada externa requerem quantidades maiores de resina em comparação às partículas maiores da camada interna do painel.

Neste trabalho, objetivou-se avaliar a qualidade de painéis aglomerados produzidos com a madeira de Melia azedarach (Cinamomo) e Pinus taeda com diferentes teores de resina ureia-formaldeído em painéis homogêneos e multicamadas.

\section{MATERIAL E MÉTODOS}

Foi utilizada, nesta pesquisa, madeira de Melia azedarach (Cinamomo) proveniente de um plantio experimental com 18,5 anos de idade, localizado em Corupá- SC. As partículas de Pinus taeda foram coletadas diretamente na linha de produção da empresa Arauco do Brasil S.A., localizada em Curitiba-PR.

A resina empregada para a colagem dos painéis foi a ureia-formaldeído, com teor de sólidos de $63,9 \%$, viscosidade Brookfield de $668 \mathrm{cP} \mathrm{e} \mathrm{pH} \mathrm{de} \mathrm{8,57.} \mathrm{O} \mathrm{sulfato}$ de amônia foi utilizado como catalisador.

Para a manufatura de painéis aglomerados em laboratório, as partículas de madeira foram geradas num picador de disco na forma de "flakes", com as dimensões nominais de $25 \mathrm{~mm}$ (comprimento) x $0,7 \mathrm{~mm}$ (espessura) e largura variável. Após a secagem ao teor de umidade médio de $3 \%$, as partículas foram re-processadas no moinho de martelo. As partículas utilizadas nos tratamentos homogêneos ( 1 e 2) e na camada externa dos demais tratamentos foram classificadas nas malhas de 8 e 30 mesh. Já, as partículas destinadas à camada interna foram classificadas entre as malhas de 6 e 8 mesh.

Os painéis foram produzidos com a formação homogênea e em multicamadas, com proporções nas camadas externas e interna de 20:60:20. A outra variável de estudo foi o teor de resina com 6,8 e 10\% de sólidos em relação ao peso seco das partículas, conforme apresentado no plano experimental da Tabela 1.

Os cálculos dos materiais foram definidos para dimensões do painel de $50 \times 50 \times 1,5 \mathrm{~cm}$ e densidade nominal de $0,75 \mathrm{~g} / \mathrm{cm}^{3}$. Os painéis foram prensados à temperatura de $160^{\circ} \mathrm{C}$, pressão específica de $40 \mathrm{kgf} / \mathrm{cm}^{2} \mathrm{e}$ tempo de prensagem de 8 minutos. Foram produzidos dois 
Tabela 1 - Plano experimental.

Table 1 - Experimental design.

\begin{tabular}{clcc}
\hline Tratamento & Espécie & Teor de adesivo & Partículas \\
\hline 1 & Pinus taeda & $8 \%$ & Homogêneo \\
2 & Meliaazedarach & $8 \%$ & Homogêneo \\
3 & Pinus taeda & $8 \%$ CI e 8\% CE & Multicamada \\
4 & Meliaazedarach & $8 \%$ CI e 8\% CE & Multicamada \\
5 & Pinus taeda & $6 \%$ CI e 8\% CE & Multicamada \\
6 & Meliaazedarach & $6 \%$ CI e 8\% CE & Multicamada \\
7 & Pinus taeda & $6 \%$ CI e 10\% CE & Multicamada \\
8 & Meliaazedarach & $6 \%$ CI e 10\% CE & Multicamada \\
\hline
\end{tabular}

CI: Camada Interna; CE: Camada Externa.

painéis por tratamento, perfazendo um total de dezesseis painéis experimentais.

Após a manufatura dos painéis, os mesmos foram conduzidos à câmara de climatização com condições ambientais controladas (T: $20 \pm 2^{\circ} \mathrm{C}$ e UR: $65 \pm 5 \%$ ), até atingirem a umidade de equilíbrio.

A qualidade dos painéis foi avaliada de acordo com a metodologia proposta pela Norma Européia EN 310, 317, 319 e 322 (EUROPEAN COMMITTEE FOR STANDARDIZATION - EN, 2003), respectivamente para flexão estática (FE), absorção de água (AA) e inchamento em espessura (IE) após 2 e 24 horas, ligação interna (LI) e resistência ao arrancamento de parafuso na superfície (RAPs) e no topo (RAPt). Foram testados para cada tratamento, 8 corpos-de-prova de FE, 18 de AA e IE, 16 de LI e 10 de RAPs e RAPt. Os valores experimentais foram comparados com os requisitos propostos pela Norma EN 312:2003 (EN, 2003).

Os dados obtidos foram avaliados estatisticamente ao nível de $95 \%$ de confiabilidade a partir do Teste de Hartley, Análise de Variância e Comparação de Médias de Tukey.

\section{RESULTADOS E DISCUSSÃO}

\subsection{Propriedades físicas dos painéis}

A massa específica média dos painéis aglomerados para os oito tratamentos avaliados foi de $0,689 \mathrm{~g} / \mathrm{cm}^{3}$. Com base na massa específica aparente média da madeira de Melia azedarach de $0,488 \mathrm{~g} / \mathrm{cm}^{3}$ e de Pinus taeda de 0,450 $\mathrm{g} / \mathrm{cm}^{3}$, os valores da razão de compactação dos painéis foi de 1.41 e 1.53, respectivamente. Esse valor está dentro da faixa de 1,3 a 1,6 conforme recomendado por Maloney (1993) e Moslemi (1974).
$\mathrm{Na}$ Tabela 2, estão apresentados os resultados de absorção de água e inchamento em espessura após 2 e 24 horas de imersão em água.

Tabela 2 - Valores médios das propriedades físicas dos painéis aglomerados.

Table 2 - Average values of physical properties of the particleboards.

\begin{tabular}{|c|c|c|c|c|}
\hline Tratamento & $\begin{array}{c}\text { AA } 2 \mathrm{~h} \\
(\%)\end{array}$ & $\begin{array}{c}\text { AA } 24 \mathrm{~h} \\
(\%)\end{array}$ & $\begin{array}{c}\text { IE } 2 \mathrm{~h} \\
(\%)\end{array}$ & $\begin{array}{c}\text { IE 24h } \\
(\%)\end{array}$ \\
\hline 1 & $\begin{array}{c}7,36 \mathrm{~d} \\
(24,65)\end{array}$ & $\begin{array}{l}20,58 \text { e } \\
(13,42)\end{array}$ & $\begin{array}{c}3,61 \mathrm{c} \\
(21,54)\end{array}$ & $\begin{array}{c}8,53 \mathrm{~d} \\
(12,60)\end{array}$ \\
\hline 2 & $\begin{array}{c}13,44 \mathrm{bc} \\
(26,76)\end{array}$ & $\begin{array}{c}33,91 \mathrm{~cd} \\
(16,88)\end{array}$ & $\begin{array}{l}4,39 \text { bc } \\
(20,42)\end{array}$ & $\begin{array}{l}9,86 \mathrm{~cd} \\
(17,69)\end{array}$ \\
\hline 3 & $\begin{array}{c}10,31 \mathrm{~cd} \\
(24,63)\end{array}$ & $\begin{array}{l}28,80 \mathrm{~d} \\
(10,42)\end{array}$ & $\begin{array}{l}4,80 \mathrm{ab} \\
(16,76)\end{array}$ & $\begin{array}{c}11,23 \mathrm{bc} \\
(16,63)\end{array}$ \\
\hline 4 & $\begin{array}{l}15,60 \mathrm{~b} \\
(24,21)\end{array}$ & $\begin{array}{c}37,10 \mathrm{bc} \\
(14,04)\end{array}$ & $\begin{array}{l}4,88 \mathrm{ab} \\
(23,24)\end{array}$ & $\begin{array}{c}12,97 \mathrm{ab} \\
(15,37)\end{array}$ \\
\hline 5 & $\begin{array}{c}11,88 \text { bc } \\
(11,24)\end{array}$ & $\begin{array}{c}33,36 \mathrm{~cd} \\
(6,29)\end{array}$ & $\begin{array}{l}4,56 \text { bc } \\
(22,94)\end{array}$ & $\begin{array}{l}14,19 \mathrm{a} \\
(17,83)\end{array}$ \\
\hline 6 & $\begin{array}{l}19,83 \mathrm{a} \\
(30,66)\end{array}$ & $\begin{array}{l}43,29 a \\
(20,84)\end{array}$ & $\begin{array}{c}5,74 \mathrm{a} \\
(28,15)\end{array}$ & $\begin{array}{c}13,21 \mathrm{ab} \\
(17,95)\end{array}$ \\
\hline 7 & $\begin{array}{c}10,59 \mathrm{~cd} \\
(37,49)\end{array}$ & $\begin{array}{c}31,98 \mathrm{~cd} \\
(19,48)\end{array}$ & $\begin{array}{l}4,55 \mathrm{bc} \\
(27,89)\end{array}$ & $\begin{array}{c}13,46 \mathrm{ab} \\
(21,27)\end{array}$ \\
\hline 8 & $\begin{array}{c}12,41 b c \\
(47,31)\end{array}$ & $\begin{array}{c}39,75 \mathrm{ab} \\
(16,08)\end{array}$ & $\begin{array}{c}4,68 \mathrm{abc} \\
(20,36)\end{array}$ & $\begin{array}{l}14,06 \mathrm{a} \\
(16,66)\end{array}$ \\
\hline
\end{tabular}

Médias seguidas de mesma letra na mesma coluna são estatisticamente iguais pelo Teste de Tukey a 95\% de confiabilidade. Valores entre parênteses referem-se ao Coeficiente de Variação.

Os valores médios de absorção de água 2 e 24 horas variaram, respectivamente, na faixa de 7,36\% a $19,83 \%$ e $20,58 \%$ a 43,29\%. Para os painéis homogêneos, os produzidos com Pinus taeda apresentaram média de absorção de água estatisticamente inferior em comparação aos painéis de Melia azedarach, para 2 e 24 horas de imersão. A mesma tendência pode ser observada para os painéis multicamadas, com maior absorção de água dos painéis de Melia azedarach.

$\mathrm{O}$ aumento do teor de resina da camada interna de $6 \%$ para $8 \%$, resultou em menor absorção de água dos painéis nos ensaios de 2 e 24 horas de imersão. Entretanto, a diferença significativa foi constatada apenas para os painéis de Melia azedarach. Por outro lado, com o aumento do teor de resina das camadas externas de $8 \%$ para $10 \%$, verificou-se uma tendência de redução na

Cerne, Lavras, v. 18, n. 3, p. 465-470, jul./set. 2012 
absorção de água 2 e 24 horas. A diferença estatística foi constatada apenas entre os painéis de Melia azedarach para absorção de água 2 horas. Esses resultados indicam que o maior recobrimento das partículas com a resina dificulta o processo de absorção de água.

$\mathrm{Na}$ comparação com os resultados apresentados na literatura, os resultados de absorção de água obtidos neste estudo foram bem inferiores aos obtidos por vários pesquisadores. Iwakiri et al. (1996) obtiveram para painéis aglomerados de Pinus taeda e Eucalyptus dunnii, valores médios de absorção de água 24 horas de $75,04 \%$ e $80,05 \%$, respectivamente. Naumann et al. (2008) encontraram para painéis aglomerados de Eucalyptus urophylla e Schizolobium amazonicum, valores médios de $97,2 \%$ e $117,9 \%$, respectivamente. No estudo realizado por Iwakiri et al. (2004), para painéis aglomerados produzidos com Grevílea robusta com densidade de 0,60 e $0,80 \mathrm{~g} / \mathrm{cm}^{3}$, os valores médios de absorção de água 24 horas obtidos foram de $42,91 \%$ e $66,59 \%$, respectivamente.

Os valores médios de inchamento em espessura 2 e 24 horas variaram respectivamente na faixa de 3,61\% a $5,74 \%$ e $8,53 \%$ a $14,19 \%$. Para os painéis homogêneos não foram constatadas diferenças estatisticamente significativas entre os painéis produzidos com Pinus taeda e Melia azedarach, tanto para 2, quanto para 24 horas de imersão. Os resultados entre as espécies foram similares também para os painéis multicamadas, com exceção do inchamento em espessura 2 horas dos painéis com $6 \%$ de resina na camada interna e $8 \%$ na camada externa.

O aumento no teor de resina da camada interna de $6 \%$ para $8 \%$, resultou em menor inchamento em espessura 24 horas dos painéis de Pinus taeda. Para todos os demais tratamentos não foram constatadas diferenças estatisticamente significativas.

Os resultados de inchamento em espessura 24 horas obtidos nesta pesquisa foram inferiores aos valores apresentados na literatura. Iwakiri et al. (1996) encontraram para painéis aglomerados de Pinus taeda e Eucalyptus dunnii, valores médios de 30,50\% e 35,09\%, respectivamente. Naumann et al. (2008) obtiveram para painéis de Eucalyptus urophylla e Schizolobium amazonicum, valores médios de $16,8 \%$ e $17,1 \%$. Num outro estudo, Iwakiri et al. (2004) encontraram para painéis aglomerados de Grevílea robusta produzidos com densidade de 0,60 e $0,80 \mathrm{~g} / \mathrm{cm}^{3}$, inchamento em espessura 24 horas de $17,71 \%$ e $20,88 \%$, respectivamente.

Cerne, Lavras, v. 18, n. 3, p. 465-470, jul./set. 2012

\subsection{Propriedades mecânicas dos painéis}

Na Tabela 3, estão apresentados os resultados de módulo de ruptura (MOR) e módulo de elasticidade (MOE) em flexão estática, ligação interna e arrancamento de parafuso na superfície e no topo.

Tabela 3 - Valores médios das propriedades mecânicas dos painéis aglomerados.

Table 3 -Average values of mechanical properties of the particleboards.

\begin{tabular}{|c|c|c|c|c|c|}
\hline \multirow{2}{*}{ Tratamento } & \multicolumn{2}{|c|}{ Flexão estática } & \multirow{2}{*}{$\begin{array}{l}\text { Ligação } \\
\text { interna } \\
(\mathrm{MPa})\end{array}$} & \multicolumn{2}{|c|}{$\begin{array}{c}\text { Arrancamento de } \\
\text { Parafuso }\end{array}$} \\
\hline & $\begin{array}{l}\text { MOR } \\
(\mathrm{MPa})\end{array}$ & $\begin{array}{l}\mathrm{MOE} \\
(\mathrm{MPa})\end{array}$ & & $\begin{array}{l}\text { Superfície } \\
\text { (N) }\end{array}$ & $\begin{array}{l}\text { Topo } \\
(\mathrm{N})\end{array}$ \\
\hline 1 & $\begin{array}{l}12,88 \mathrm{~b} \\
(12,89)\end{array}$ & $\begin{array}{l}1.948 \mathrm{c} \\
(13,03)\end{array}$ & $\begin{array}{c}0,86 \mathrm{c} \\
(12,58)\end{array}$ & $\begin{array}{l}1.031 \mathrm{~b} \\
(14,94)\end{array}$ & $\begin{array}{c}905 \mathrm{~b} \\
(26,23)\end{array}$ \\
\hline 2 & $\begin{array}{l}19,59 \mathrm{a} \\
(15,66)\end{array}$ & $\begin{array}{c}2.265 \mathrm{abc} \\
(14,31)\end{array}$ & $\begin{array}{c}1,55 \mathrm{a} \\
(30,95)\end{array}$ & $\begin{array}{l}1.900 \mathrm{a} \\
(19,17)\end{array}$ & $\begin{array}{l}1.615 \mathrm{a} \\
(23,52)\end{array}$ \\
\hline 3 & $\begin{array}{c}12,71 \mathrm{~b} \\
(7,01)\end{array}$ & $\begin{array}{c}2.018 \mathrm{bc} \\
(5,01)\end{array}$ & $\begin{array}{l}0,64 \mathrm{~cd} \\
(19,15)\end{array}$ & $\begin{array}{l}1.189 \mathrm{~b} \\
(13,35)\end{array}$ & $\begin{array}{l}952 \mathrm{~b} \\
(13,79)\end{array}$ \\
\hline 4 & $\begin{array}{l}19,07 \mathrm{a} \\
(14,50)\end{array}$ & $\begin{array}{c}2.426 \mathrm{ab} \\
(14,23)\end{array}$ & $\begin{array}{l}1,40 \mathrm{ab} \\
(10,86)\end{array}$ & $\begin{array}{l}1.826 \mathrm{a} \\
(16,98)\end{array}$ & $\begin{array}{l}1.742 \mathrm{a} \\
(23,26)\end{array}$ \\
\hline 5 & $\begin{array}{l}11,85 \mathrm{~b} \\
(12,58)\end{array}$ & $\begin{array}{l}1.895 \mathrm{c} \\
(12,34)\end{array}$ & $\begin{array}{c}0,53 \mathrm{~d} \\
(14,84)\end{array}$ & $\begin{array}{l}1.079 \mathrm{~b} \\
(16,32)\end{array}$ & $\begin{array}{l}900 \mathrm{~b} \\
(18,56)\end{array}$ \\
\hline 6 & $\begin{array}{l}19,24 \mathrm{a} \\
(15,22)\end{array}$ & $\begin{array}{c}2.406 \mathrm{ab} \\
(13,33)\end{array}$ & $\begin{array}{l}1,43 \mathrm{ab} \\
(13,21)\end{array}$ & $\begin{array}{l}1.679 \mathrm{a} \\
(22,73)\end{array}$ & $\begin{array}{l}1.502 \mathrm{a} \\
(17,91)\end{array}$ \\
\hline 7 & $\begin{array}{l}11,55 \mathrm{~b} \\
(17,02)\end{array}$ & $\begin{array}{l}1.945 \mathrm{c} \\
(14,41)\end{array}$ & $\begin{array}{c}0,43 \mathrm{~d} \\
(14,87)\end{array}$ & $\begin{array}{l}1.032 \mathrm{~b} \\
(19,26)\end{array}$ & $\begin{array}{l}754 \mathrm{~b} \\
(24,92)\end{array}$ \\
\hline 8 & $\begin{array}{l}20,15 \mathrm{a} \\
(15,80)\end{array}$ & $\begin{array}{l}2.488 \mathrm{a} \\
(13,60)\end{array}$ & $\begin{array}{l}1,29 \mathrm{~b} \\
(12,52)\end{array}$ & $\begin{array}{l}1.820 \mathrm{a} \\
(20,54)\end{array}$ & $\begin{array}{l}1.713 \mathrm{a} \\
(27,17)\end{array}$ \\
\hline
\end{tabular}

Médias seguidas de mesma letra na mesma coluna são estatisticamente iguais pelo Teste de Tukey a 95\% de confiabilidade. Valores entre parênteses referem-se ao Coeficiente de Variação.

Os valores médios de módulo de ruptura (MOR) variaram na faixa de $11,55 \mathrm{MPa}$ a 20,15 MPa. Tanto para os painéis homogêneos, quanto para os multicamadas, os painéis produzidos com madeira de Melia azedarach apresentaram valor médio de MOR estatisticamente superior em relação aos painéis de Pinus taeda. Todos os painéis de Melia azedarach apresentaram valores médios de MOR superiores ao requisito mínimo da norma européia EN 312:2003 (EN, 2003) de $13 \mathrm{MPa}$. Entretanto, o mesmo não ocorreu com todos os painéis de Pinus taeda. 
Cabe ressaltar que, os valores superiores de MOR obtidos para os painéis de Melia azedarach, podem ser resultantes da melhor adesão entre as partículas dessa espécie, uma vez que a razão de compactação dos painéis de Melia azedarach, de 1,41 foi inferior ao obtido para painéis de Pinus taeda, que foi de 1,53.

Com relação às variações nos teores de resina dos painéis multicamadas, não foram constatadas diferenças estatisticamente significativas entre os tratamentos, com o aumento do teor de resina da camada interna de $6 \%$ para $8 \%$, e das camadas externas de $8 \%$ para $10 \%$, tanto para painéis de Pinus taeda, quanto para os de Melia azedarach.

Os resultados de MOR obtidos para os painéis de Melia azedarach foram superiores aos valores referenciais apresentados na literatura. Iwakiri et al. (1996) encontraram para painéis aglomerados de Pinus taeda e Eucalyptus dunnii, valores médios de MOR de 16,94 MPa e 15,86 MPa, respectivamente. Cabral et al. (2007) obtiveram para painéis aglomerados produzidos com maravalhas de Eucalyptus grandis, mistura de Eucalyptus urophylla e Pinus sp, mistura de Eucalyptus cloeziana e Pinus sp, valores médios de MOR de 15,52 MPa, 16,77 MPa e 17,92 MPa, respectivamente.

Os valores médios de módulo de elasticidade (MOE) variaram na faixa de $1.895 \mathrm{MPa}$ a $2.488 \mathrm{MPa}$. Os painéis homogêneos produzidos com madeira de Pinus taeda e Melia azedarach não apresentaram diferenças significativas entre si ao nível de probabilidade de $95 \%$. Já, os painéis multicamadas de Melia azedarach apresentaram maiores valores médios de MOE em comparação aos painéis de Pinus taeda. Entretanto, entre os tratamentos 3 e 4 , com $8 \%$ de resina nas camadas internas e externas, a diferença entre as médias não foi estatisticamente significativa. Os valores médios de MOE obtidos para todos os tratamentos atendem ao requisito mínimo da norma européia EN 312:2003 (EN, 2003) de $1.600 \mathrm{MPa}$.

O aumento no teor de resina de $6 \%$ para $8 \%$ nas camadas internas e de $8 \%$ para $10 \%$ nas camadas externas dos painéis não afetaram os resultados de $\mathrm{MOE}$ de forma estatisticamente significativa ao nível de probabilidade de $95 \%$.

Os resultados de MOE obtidos neste estudo são satisfatórios em comparação aos dados apresentados na literatura. Naumann et al. (2008) obtiveram para painéis de Eucalyptus urophylla e Schizolobium amazonicum, valores médios de MOE de $734 \mathrm{MPa}$ e $1.873 \mathrm{MPa}$, respectivamente. Iwakiri et al. (1996) encontraram para painéis de Pinus taeda e Eucalyptus dunnii, valores médios de MOE de 2.269 MPa e 2.312 MPa, respectivamente.
Os valores médios de ligação interna variaram na faixa de 0,43 MPa a 1,55 MPa. Tanto para os painéis homogêneos, quanto para os multicamadas, os painéis produzidos com madeira de Melia azedarach apresentaram valores médios de ligação interna estatisticamente superiores em comparação aos painéis de Pinus taeda. Analisando os resultados obtidos para os painéis multicamadas de Pinus taeda e Melia azedarach, observase que o aumento no teor de resina da camada interna de $6 \%$ para $8 \%$ não contribuiu de forma estatisticamente significativa para o incremento nos valores médios de ligação interna. Esse resultado é vantajoso sobre o ponto de vista econômico, tendo em vista a possibilidade de uso de menor quantidade de resina no processo produtivo.

Os valores médios de ligação interna obtidos para todos os tratamentos avaliados atendem ao requisito mínimo da norma européia EN 312:2003 (EN, 2003) de 0,35 MPa. Pode-se destacar também os elevados valores de ligação interna obtidos para os painéis de Melia azedarach, indicando o alto potencial desta espécie para produção de painéis aglomerados.

Com relação aos resultados apresentados na literatura, Iwakiri et al. (1996) obtiveram para painéis de Pinus taeda e Eucalyptus dunnii, valores médios de 1,10 e 0,82 MPa, respectivamente. Já, Colli et al. (2010) encontraram para painéis aglomerados produzidos com Schizolobium amazonicum valor médio de ligação interna de 0,22 MPa. Portanto, os resultados de ligação interna obtidos neste estudo estão compatíveis com os valores obtidos para várias espécies de madeiras pesquisadas.

Os valores médios de arrancamento de parafusos na superfície e topo variaram na faixa de $1.031 \mathrm{a}$ $1.900 \mathrm{MPa}$ e de 754 a $1.742 \mathrm{MPa}$, respectivamente. Os resultados indicam que para todos os tratamentos, os painéis de Melia azedarach apresentaram valores médios de arrancamento de parafuso superfície e topo, estatisticamente superiores em comparação aos painéis de Pinus taeda.

$\mathrm{O}$ aumento no teor de resina da camada interna de $6 \%$ para $8 \%$, e das camadas externas de $8 \%$ para $10 \%$, não contribuiram de forma estatisticamente significativa para o incremento nos valores médios de resistência ao arrancamento de parafuso, tanto para os painéis de Pinus taeda, quanto para os de Melia azedarach.

Os resultados obtidos nesta pesquisa estão compatíveis com os encontrados por Trianoski (2010), para painéis aglomerados produzidos com madeiras de Acrocarpus fraxinifolius, Grevilea robusta, Schizolobium parahyba e Toona ciliata, cujos valores médios de

Cerne, Lavras, v. 18, n. 3, p. 465-470, jul./set. 2012 
resistência ao arrancamento de parafuso na superfície foram de $1.503,920,1.244$ e $1.705 \mathrm{MPa}$, e, para arrancamento de parafuso no topo de 1.571, 735, $1.244 \mathrm{e}$ $1.493 \mathrm{MPa}$, respectivamente.

\section{CONCLUSÕES}

Os painéis produzidos com a madeira de Melia azedarach apresentaram melhores resultados de propriedades físico-mecânicas em comparação aos painéis de Pnus taeda.

Os resultados das propriedades dos painéis obtidos tanto para o Pinus taeda, quanto para o Melia azedarach, estão dentro da faixa de valores citados na literatura e atendem aos requisitos mínimos da norma Européia EN 312:2003 (EN, 2003).

O aumento no teor de resina da camada interna e das camadas externas teve efeitos positivos sobre as propriedades de absorção de água e inchamento em espessura dos painéis. Por outro lado, as propriedades mecânicas não foram afetadas pelo aumento no teor de resina.

Os resultados desta pesquisa indicam um grande potencial da espécie Melia azedarach para a produção de painéis aglomerados.

\section{AGRADECIMENTOS}

Os autores expressam seus agradecimentos a Battistella Florestal, pelo uso do plantio experimental de Melia azedarach e à Arauco do Brasil, pela doação das partículas de Pinus taeda, resina e parafina.

\section{REFERÊNCIAS}

\section{BANCO NACIONAL DE DESENVOLVIMENTO} ECONÔMICO E SOCIAL. Painéis de madeira no Brasil: atualidades e perspectivas: relatório técnico. Rio de Janeiro, 2008. 156 p.

CABRAL, C. P.; VITAL, B. R.; LUCIA, R. M. D.; PIMENTA, A. S. Propriedades de chapas de aglomerado confeccionadas com mistura de partículas de Eucalyptus spp and Pinus elliottii. Revista Árvore, Viçosa, v. 31, n. 5, p. 897-905, set./ out. 2007.

CENTRO AGRONÔMICO TROPICAL DE IVESTIGACION Y ENSEÑANZA. Silvicultura de espécies promissoras para produccion de lema em America Central. Turrialba, 1986. 219 p. (Informe Técnico, 86).

Cerne, Lavras, v. 18, n. 3, p. 465-470, jul./set. 2012
COLLI, A.; VITAL, B. R.; CARNEIRO, A. C. O.; SILVA, J. C.; CARVALHO, A. N. M. L.; DELLA LUCIA, R. M. Propriedades de chapas fabricadas com partículas de madeira de paricá (Schizolonium amazonicum Huber ex.Ducke) e fibras de coco (Cocos nucifera L.). Revista Árvore, Viçosa, v. 34, n. 2, p. 333-338, mar./abr. 2010.

\section{EUROPEAN COMMITTEE FOR STANDARDIZATION.} Normas EN. Dordrecht, 2003.

IWAKIRI, S.; LATORRACA, J. V. F.; SILVA, D. A.; GABARDO, J. L.; KLITZKE, R. J.; FOFANO, A.; FABROWSKI, F.; INTERANMENSE, M. T. Produção de chapas de partículas de Madeira aglomerada de Pinus elliottii (Engelm) e Eucalyptus dunnii (Maid). Ciências Agrárias, Teresina, v. 15, n. 1, p. 33-41, 1996.

IWAKIRI, S.; SHIMIZU, J.; SILVA, J. C.; DEL MENEZZI, C. H. S.; PUEHRINGER, C. A.; VENSON, I.; LAROCA, C. Produção de painéis de madeira aglomerada de Grevilea robusta A. Cunn. Ex R. Br. Revista Árvore, Viçosa, v. 28, n. 6, p. 56-60, nov./dez. 2004.

MALONEY, T. M. Modern particleboard and dry-process fiberboard manufacturing. San Francisco: M. Freeman, 1993. $689 \mathrm{p}$.

MARRA, F. S. Technology of wood bonding: principles in practice. New York: V. N. Reinhold, 1992. 453 p.

MOSLEMI, A. A. Particleboard: materials. London: Southern Illinois University, 1974. v. 1, 244 p.

NAUMANN, R. B.; VITAL, B. R.; CARNEIRO, A. C. O.; DELLA LUCIA, R. M.; SILVA, J. C.; CARVALHO, A. M. M. L.; COLLI, A. Propriedades de chapas fabricadas com partículas de madeira de Eucalyptus urophylla S.T.Blake e Schizolobium amazonicum Herb. Revista Árvore, Viçosa, v. 32, n. 6, p. 1143-1150, nov./dez. 2008.

TRIANOSKI, R. Avaliação do potencial de espécies florestais alternativas de rápido crescimento para produção de painéis de madeira aglomerada. 2010. 260 p. Dissertação (Mestrado em Ciências Florestais) - Universidade Federal do Paraná, Curitiba, 2010.

VENSON, I. Estúdio de lãs propriedades de la madera de Melia azedarach Linn. 2003. 119 p. Dissertação (Maestro em Ciência de Productos Forestalis) - Universidad de Guadalajara, Guadaljara, 2003. 